\title{
QUASINORMALITY OF ORDER 1 FOR FAMILIES OF MEROMORPHIC FUNCTIONS
}

\author{
Shahar Nevo ${ }^{1}$ AND Xuecheng Pang ${ }^{2}$
}

\begin{abstract}
Let $\mathscr{F}$ be a family of functions meromorphic on the plane domain $D$, all of whose zeros are multiple. Suppose that $f^{(k)}(z) \neq 1$ for all $f \in \mathscr{F}$ and $z \in D$. Then if $\mathscr{F}$ is quasinormal on $D$, it is quasinormal of order 1 there.
\end{abstract}

\section{Introduction}

This paper continues our study of the order of quasinormality of families of meromorphic functions on plane domains, all of whose zeros are multiple, initiated in [6].

Recall that a family $\mathscr{F}$ of functions meromorphic on a plane domain $D \subset \boldsymbol{C}$ is said to be quasinormal on $D$ [2] if from each sequence $\left\{f_{n}\right\} \subset \mathscr{F}$ one can extract a subsequence $\left\{f_{n_{k}}\right\}$ which converges locally uniformly with respect to the spherical metric on $D \backslash E$, where the set $E$ (which may depend on $\left\{f_{n_{k}}\right\}$ ) has no accumulation point in $D$. If $E$ can always be chosen to satisfy $|E| \leq v, \mathscr{F}$ is said to quasinormal of order $v$ on $D$. Thus a family is quasinormal of order 0 on $D$ if and only if it is normal on $D$. The family $\mathscr{F}$ is said to (quasi)normal at $z_{0} \in D$ if it is (quasi)normal on some neighborhood of $z_{0}$; thus $\mathscr{F}$ is quasinormal on $D$ if and only if it is quasinormal at each point $z \in D$. On the other hand, $\mathscr{F}$ fails to be quasinormal of order $v$ on $D$ precisely when there exist points $z_{1}, z_{2}, \ldots, z_{v+1}$ in $D$ and a sequence $\left\{f_{n}\right\} \subset \mathscr{F}$ such that no subsequence of $\left\{f_{n}\right\}$ is normal at $z_{j}$, $j=1,2, \ldots, v+1$.

In [6], we proved

THEOREM A. Let $\mathscr{F}$ be a quasinormal family of meromorphic functions on $D$, all of whose zeros are multiple. If for any $f \in \mathscr{F}, f^{\prime}(z) \neq 1$ for $z \in D$, then $\mathscr{F}$ is quasinormal of order 1 on $D$.

Here we extend this result to derivatives of arbitrary order.

2000 Mathematics Subject Classification: 30D45.

${ }^{1}$ Research supported by the German-Israeli Foundation for Scientific Research and Development, G.I.F. Grant No. G-643-117.6/1999.

${ }^{2}$ Research supported by the NNSF of China Approved No. 10271122.

Received October 15, 2003; revised March 4, 2004. 
THeOREM. Let $k \geq 1$ be an integer. Let $\mathscr{F}$ be a quasinormal family of meromorphic functions on $D$, all of whose zeros have multiplicity at least $k+1$. If for any $f \in \mathscr{F}, f^{(k)}(z) \neq 1$ for $z \in D$, then $\mathscr{F}$ is quasinormal of order 1 on $D$.

Corollary. Let $k$ and $M$ be positive numbers. Let $\mathscr{F}$ be a family of meromorphic functions on $D$, all of whose zeros have multiplicity at least $k+1$. Suppose that each $f \in \mathscr{F}$ has at most $M$ zeros on $D$ and that $f^{(k)}(z) \neq 1$ on D. Then $\mathscr{F}$ is quasinormal of order 1 on $D$.

Indeed, it follows easily from Lemma 2 below that $\mathscr{F}$ is quasinormal of order no greater than $M$, so the hypotheses of our Theorem are satisfied. That $\mathscr{F}$ need not be normal on $D$ is shown by the following example.

Example 1. Let $D=\{z:|z|<1\}$ and $\mathscr{F}=\left\{f_{\alpha}\right\}$, where

$$
f_{\alpha}(z)=\frac{(z-\alpha /(k+1))^{k+1}}{k !(z-\alpha)}=\frac{1}{k !} z^{k}+P_{k-2}(z)+\frac{A}{z-\alpha}, \quad \alpha \in C \backslash\{0\},
$$

where $P_{k-2}$ is a polynomial of degree $k-2$ and $A=(1 / k !)(k /(k+1))^{k+1} \alpha^{k+1} \neq 0$. Then all zeros of $f_{\alpha}$ have multiplicity at least $k+1$ and $f_{\alpha}^{(k)}(z) \neq 1$. However, $f_{\alpha}$ takes on the values 0 and $\infty$ in any fixed neighborhood of 0 if $\alpha$ is sufficiently small, so $\mathscr{F}$ fails to be normal at 0 .

Acknowledgment. This work was done while the second author (X. P.) held a research position at Bar-Ilan University. He thanks the Mathematics Department of that institution for its warm hospitality. Both authors thank Professor Lawrence Zalcman for his interest and helpful comments.

\section{Notation and preliminary results}

Let us set some notation. Throughout, $k$ is a positive integer. We denote by $\Delta$ the open unit disc in $\boldsymbol{C}$. For $z_{0} \in \boldsymbol{C}$ and $r>0, \Delta\left(z_{0}, r\right)=\left\{z:\left|z-z_{0}\right|<r\right\}$ and $\Delta^{\prime}\left(z_{0}, r\right)=\left\{z: 0<\left|z-z_{0}\right|<r\right\}$. We write $f_{n} \stackrel{\chi}{\Rightarrow} f$ on $D$ to indicate that the sequence $\left\{f_{n}\right\}$ converges to $f$ in the spherical metric uniformly on compact subsets of $D$ and $f_{n} \Rightarrow f$ on $D$ if the convergence is in the Euclidean metric.

We require the following known results.

LEMma 1. Let $\mathscr{F}$ be a family of functions meromorphic on $\Delta$, all of whose zeros have multiplicity at least $k$, and suppose that there exists $A \geq 1$ such that $\left|f^{(k)}(z)\right| \leq A$ whenever $f(z)=0$. Then if $\mathscr{F}$ is not normal at $z_{0}$, there exist, for each $0 \leq \alpha \leq k$,

a) points $z_{n} \in \Delta, z_{n} \rightarrow z_{0}$;

b) functions $f_{n} \in \mathscr{F}$; and

c) positive numbers $\rho_{n} \rightarrow 0$ 
such that $\rho_{n}^{-\alpha} f_{n}\left(z_{n}+\rho_{n} \zeta\right)=g_{n}(\zeta) \stackrel{\chi}{\Rightarrow} g(\zeta)$ on $\boldsymbol{C}$, where $g$ is a nonconstant meromorphic function on $\boldsymbol{C}$, all of whose zeros have multiplicity at least $k$, such that $g^{\#}(\zeta) \leq g^{\#}(0)=k A+1$. In particular, $g$ has order at most 2 .

Here, as usual, $g^{\#}(\zeta)=\left|g^{\prime}(\zeta)\right| /\left(1+|g(\zeta)|^{2}\right)$ is the spherical derivative.

This is the local version of [7, Lemma 2] (cf. [4, Lemma 1], [10, pp. 216217]). The proof consists of a simple change of variable in the result cited from [7]; cf. [5, pp. 299-300].

Lemma 2. Let $\mathscr{F}$ be a family of functions meromorphic on $D$ and let $k \geq 1$ be an integer. If for each $f \in \mathscr{F}$ and $z \in D, f(z) \neq 0$ and $f^{(k)}(z) \neq 1$, then $\mathscr{F}$ is normal on $D$.

This is a well-known result of $\mathrm{Gu}[3]$.

Lemma 3. Let $\mathscr{F}$ be a family of functions meromorphic on $D$, all of whose zeros have multiplicity at least $k+1$ and all of whose poles are multiple. If for each $f \in \mathscr{F}, f^{(k)}(z) \neq 1, z \in D$, then $\mathscr{F}$ is normal on $D$.

This is Theorem 5 in [9].

Lemma 4. Let $f$ be a nonconstant meromorphic function of finite order on $\boldsymbol{C}$, all of whose zeros have multiplicity at least $k+1$. If $f^{(k)}(z) \neq 1$ on $\boldsymbol{C}$, then

$$
f(z)=\frac{1}{k !} \frac{(z-a)^{k+1}}{z-b}
$$

for some $a$ and $b(\neq a)$ in $\boldsymbol{C}$.

This follows from Lemmas 6 and 8 of [9].

\section{Auxiliary lemmas}

The proof of the theorem proceeds by a number of intermediate results.

Lemma 5. Let $\left\{a_{j}\right\}$ be a sequence in $\Delta$ which has no accumulation points in $\Delta$. Let $\left\{f_{n}\right\}$ be a sequence of functions meromorphic on $\Delta$, all of whose zeros have multiplicity at least $k+1$, such that $f_{n}^{(k)}(z) \neq 1$ for all $n$ and all $z \in \Delta$. Suppose that

Then

(a) no subsequence of $\left\{f_{n}\right\}$ is normal at $a_{1}$;

(b) there exists $\delta>0$ such that each $f_{n}$ has a single (multiple) zero on $\Delta\left(a_{1}, \delta\right)$; and $\gamma$

(c) $f_{n} \stackrel{\chi}{\Rightarrow} f$ on $\Delta \backslash\left\{a_{j}\right\}_{j=1}^{\infty}$.

(d) there exists $\eta_{0}>0$ such that for each $0<\eta<\eta_{0}$, $f_{n}$ has a single simple pole on $\Delta\left(a_{1}, \eta\right)$ for all sufficiently large $n$; and

(e) $f(z)=\left(z-a_{1}\right)^{k} / k$ ! 
Proof. It suffices to prove that each subsequence of $\left\{f_{n}\right\}$ has a subsequence which satisfies (d) and (e). So suppose we have a subsequence of $\left\{f_{n}\right\}$, which (to avoid complication in notation) we again call $\left\{f_{n}\right\}$.

Since $\left\{f_{n}\right\}$ is not normal at $a_{1}$, it follows from Lemma 1 that we can extract a subsequence (which, renumbering, we continue to call $\left\{f_{n}\right\}$ ), points $z_{n} \rightarrow a_{1}$, and positive numbers $\rho_{n} \rightarrow 0$ such that

$$
g_{n}(\zeta)=\frac{f_{n}\left(z_{n}+\rho_{n} \zeta\right)}{\rho_{n}^{k}} \stackrel{\chi}{\Rightarrow} g(\zeta) \quad \text { on } \boldsymbol{C}
$$

where $g$ is a nonconstant meromorphic function of finite order on $\boldsymbol{C}$, all of whose zeros have multiplicity at least $k+1$. Since $g_{n}^{(k)}(\zeta)=f_{n}^{(k)}\left(z_{n}+\rho_{n} \zeta\right) \neq 1$ and $g_{n}^{(k)} \Rightarrow g^{(k)}$ on the complement of the poles of $g$, either $g^{(k)} \neq 1$ or $g^{(k)} \equiv 1$, by Hurwitz' Theorem. In the latter case, $g$ is a polynomial of degree $k$ and therefore does not have zeros of multiplicity at least $k+1$. Thus $g^{(k)}(\zeta) \neq 1$ on $\boldsymbol{C}$; so by Lemma 4,

$$
g(\zeta)=\frac{1}{k !} \frac{(\zeta-a)^{k+1}}{\zeta-b}
$$

for distinct complex numbers $a$ and $b$. It now follows from the argument principle that there exist sequences $\xi_{n} \rightarrow a$ and $\eta_{n} \rightarrow b$ such that, for sufficiently large $n, g_{n}\left(\xi_{n}\right)=0$ and $g_{n}\left(\eta_{n}\right)=\infty$. Thus, writing $z_{n, 0}=z_{n}+\rho_{n} \xi_{n}, z_{n, 1}=z_{n}+\rho_{n} \eta_{n}$, we have $z_{n, j} \rightarrow a_{1}(j=0,1), f_{n}\left(z_{n, 0}\right)=0$ and $f_{n}\left(z_{n, 1}\right)=\infty$.

Let us now assume that (d) has been shown to hold. It follows from Lemma 3 that the pole of $f_{n}$ at $z_{n, 1}$ is simple. The limit function $f$ from (c) is either meromorphic on $\Delta \backslash\left\{a_{j}\right\}_{j=1}^{\infty}$ or identically infinite there. Suppose first that it is meromorphic on $\Delta \backslash\left\{a_{j}\right\}_{j=1}^{\infty}$. Then there exists $\delta_{0}>0$ such that $f$ has no poles on $\Gamma=\left\{z:\left|z-a_{1}\right|=\delta_{0}\right\}$ and $f_{n}^{(k)}$ converges uniformly to $f^{(k)}$ on $\Gamma$. We claim that $f^{(k)} \equiv 1$ on $\Delta^{\prime}\left(a_{1}, \delta_{0}\right)$. Indeed, otherwise by Hurwitz' Theorem, $f^{(k)} \neq 1$. Now $1 /\left(f_{n}^{(k)}-1\right)$ is analytic on $\Delta\left(a_{1}, \delta_{0}\right)$ and converges uniformly on $\Gamma$ to $1 /\left(f^{(k)}-1\right)$. By the maximum principle, $1 /\left(f_{n}^{(k)}-1\right)$ converges uniformly on $\Delta\left(a_{1}, \delta_{0}\right)$, so $\left\{f_{n}^{(k)}\right\}$ is normal at $a_{1}$. However, since $f_{n}^{(k)}\left(z_{n, 0}\right)=0$ and $f_{n}^{(k)}\left(z_{n, 1}\right)=\infty$ and $z_{n, j} \rightarrow a_{1}(j=0,1),\left\{f_{n}^{(k)}\right\}$ is not equicontinuous at $a_{1}$, a contradiction.

Thus $f$ has no poles on $\Delta^{\prime}\left(a_{1}, \delta_{0}\right)$ and $f_{n}^{(k)} \Rightarrow 1$ on $\Delta^{\prime}\left(a_{1}, \delta_{0}\right)$. We claim now that for every $0 \leq i \leq k$

$$
f_{n}^{(k-i)}(z) \Rightarrow \frac{\left(z-a_{1}\right)^{i}}{i !} \text { on } \Delta^{\prime}\left(a_{1}, \delta_{0}\right)
$$

We have already proved this for $i=0$.

We continue by induction. Suppose that (3) holds for $i=j$ and let $i=j+1$. For $z, z_{0} \in \Delta^{\prime}\left(a_{1}, \delta_{0}\right)$, we have

$$
f_{n}^{(k-(j+1))}(z)-f_{n}^{(k-(j+1))}\left(z_{0}\right)=\int_{z_{0}}^{z} f_{n}^{(k-j)}(\zeta) d \zeta .
$$


By the induction assumption, the last term tends to $\left(z-a_{1}\right)^{j+1} /(j+1)$ !$\left(z_{0}-a_{1}\right)^{j+1} /(j+1) !$; thus

$$
f_{n}^{(k-(j+1))}(z) \Rightarrow \frac{\left(z-a_{1}\right)^{j+1}}{(j+1) !}+\beta\left(z_{0}\right),
$$

where $\beta\left(z_{0}\right)=\lim _{n \rightarrow \infty}\left[f_{n}^{(k-(j+1))}\left(z_{0}\right)-\left(z_{0}-a_{1}\right)^{j+1} /(j+1) !\right]$.

We now show that $\beta\left(z_{0}\right)=0$. If not, take $r$ such that $0<r<$ $\min \left\{\left|(j+1) ! \beta\left(z_{0}\right)\right|^{1 /(j+1)}, \delta_{0}\right\}$. For large enough $n$, we have

$$
\frac{1}{2 \pi i} \int_{\left|\zeta-a_{1}\right|=r} \frac{f_{n}^{(k-j)}(\zeta)}{f_{n}^{(k-(j+1))}(\zeta)} d \zeta=\frac{1}{2 \pi i} \int_{\left|\zeta-a_{1}\right|=r} \frac{\left(\zeta-a_{1}\right)^{j} / j !}{\left(\zeta-a_{1}\right)^{j+1} /(j+1) !+\beta\left(z_{0}\right)} d \zeta
$$

Now the right hand term is zero, since the zeros of $\left(\zeta-a_{1}\right)^{j+1} /(j+1) !+\beta\left(z_{0}\right)$ are outside $\Delta\left(a_{1}, r\right)$. By condition (d), the number of poles in $\Delta\left(a_{1}, \delta_{0}\right)$ of $f_{n}^{(k-(j+1))}$ in $(4)$ is $k-(j+1)+1=k-j$, counting multiplicities.

As for the number of zeros, without loss of generality, we may assume $b=0$ in (2). Then $a \neq 0$, and we have

$$
\begin{aligned}
g(\zeta) & =\frac{1}{k !} \frac{1}{\zeta}\left[\zeta^{k+1}-(k+1) a \zeta^{k}+\cdots+(-1)^{k}\left(\begin{array}{c}
k+1 \\
k
\end{array}\right) z^{k} \zeta+(-1)^{k+1} a^{k+1}\right] \\
& =\frac{1}{k !}\left[\zeta^{k}-(k+1) a \zeta^{k-1}+\cdots+\frac{(-1)^{k+1} a^{k+1}}{\zeta}\right] .
\end{aligned}
$$

Hence, for each $0 \leq i \leq k, g^{(i)}(\zeta)$ has exactly $k+1$ zeros in $\boldsymbol{C}$, counting multiplicities. Thus by (1), for large enough $n, f_{n}^{(i)}(z)$ has at least $k+1$ zeros in $\Delta\left(a_{1}, \delta_{0}\right)$. We then get by the argument principle that the left hand term in (4) is at least $k+1-(k-j)=j+1$, and we have a contradiction. Thus $\beta\left(z_{0}\right)=0$, and (3) is proved. Take $i=k$ in (3) to get assertion (e).

Suppose now that $f \equiv \infty$ on $\Delta \backslash\left\{a_{j}\right\}_{j=1}^{\infty}$. Let

$$
F_{n}(z)=f_{n}(z) \frac{z-z_{n, 1}}{\left(z-z_{n, 0}\right)^{k+1}} .
$$

By (b), $F_{n}(z) \neq 0$ on $\Delta\left(a_{1}, \delta\right)$. Applying the maximum principle to the sequence $\left\{1 / F_{n}\right\}$ of analytic functions, we see that $F_{n} \Rightarrow \infty$ on $\Delta\left(a_{1}, \delta\right)$. We have

$$
\begin{aligned}
\frac{f_{n}\left(z_{n}+\rho_{n} \zeta\right)}{\rho_{n}^{k}} & =\frac{F_{n}\left(z_{n}+\rho_{n} \zeta\right)}{\rho_{n}^{k}} \frac{\left(\rho_{n} \zeta+z_{n}-z_{n, 0}\right)^{k+1}}{\left(\rho_{n} \zeta+z_{n}-z_{n, 1}\right)} \\
& =F_{n}\left(z_{n}+\rho_{n} \zeta\right) \frac{\left(\zeta-\xi_{n}\right)^{k+1}}{\zeta-\eta_{n}} .
\end{aligned}
$$

It follows from (1), (2), and (5) that $F_{n}\left(z_{n}+\rho_{n} \zeta\right) \rightarrow 1$, which contradicts $F_{n} \Rightarrow \infty$ near $a_{1}$. Thus the posssibility $f \equiv \infty$ may be ruled out.

We have shown that when (d) obtains, (e) does as well. Now let us show that (d) must hold. Suppose not. Then, taking a subsequence and renum- 
bering, we may assume that on any neighborhood of $a_{1}, f_{n}$ has at least two poles for sufficiently large $n$. Keeping the notation established above, let $z_{n, 2} \neq z_{n, 1}$ be such that $f_{n}\left(z_{n, 2}\right)=\infty$ and $f_{n}$ has no poles in $\Delta^{\prime}\left(z_{n, 1},\left|z_{n, 1}-z_{n, 2}\right|\right)$. Write $z_{n, 2}=$ $z_{n}+\rho_{n} \eta_{n}^{*}$. Then $z_{n, 2} \rightarrow a_{1}$ but $\eta_{n}^{*} \rightarrow \infty$ since the right hand side of (2) has but a single simple pole. Set

$$
G_{n}(\zeta)=\frac{f_{n}\left(z_{n, 1}+\left(z_{n, 2}-z_{n, 1}\right) \zeta\right)}{\left(z_{n, 2}-z_{n, 1}\right)^{k}} .
$$

Since $z_{n, 2}-z_{n, 1} \rightarrow 0, G_{n}(\zeta)$ is defined for any $\zeta \in \boldsymbol{C}$ if $n$ is sufficiently large; and $G_{n}^{(k)}(\zeta) \neq 1$. Note that $G_{n}(1)=\infty$. Also,

$$
G_{n}(0)=\infty \quad G_{n}\left(\frac{z_{n, 0}-z_{n, 1}}{z_{n, 2}-z_{n, 1}}\right)=0
$$

and

$$
\frac{z_{n, 0}-z_{n, 1}}{z_{n, 2}-z_{n, 1}}=\frac{\xi_{n}-\eta_{n}}{\eta_{n}^{*}-\eta_{n}} \rightarrow 0,
$$

so $\left\{G_{n}\right\}$ is not normal at 0 . On the other hand, for $n$ sufficiently large, $G_{n}$ has only a single zero (which tends to 0 as $n \rightarrow \infty$ ) on any compact subset of $\boldsymbol{C}$. Since $G_{n}^{\prime}(\zeta) \neq 1$, it follows from Lemma 2 that $\left\{G_{n}\right\}$ is normal on $C \backslash\{0\}$. Taking a subsequence and renumbering, we may assume that $G_{n} \stackrel{\chi}{\Rightarrow} G$ on $C \backslash\{0\}$. Since $G_{n}$ has only a single pole on $\Delta$, conditions (a), (b), (c), and (d) hold for the sequence $\left\{G_{n}\right\}$ (defined, say, on $\left.\Delta(0,2)\right)$ with $a_{1}=0$ and $\delta=1$. Thus, by the first part of the proof, $G(\zeta)=\zeta^{k} / k !$. But this contradicts $G(1)=\infty$. This completes the proof of Lemma 5 .

Definition. Let $z_{1}, z_{2} \in \boldsymbol{C}$ and put $\tilde{z}=\left(z_{1}+z_{2}\right) / 2$. We say that $\left(z_{1}, z_{2}\right)$ is a $k$-nontrivial pair of zeros of $f$ if

(i) $f\left(z_{1}\right)=f\left(z_{2}\right)=0$ and

(ii) there exists $z_{3}$ such that $\left|z_{3}-\tilde{z}\right|<\left|z_{1}-z_{2}\right|$ and $\left|f^{\prime}\left(z_{3}\right)\right| /\left|z_{1}-z_{2}\right|^{k-1}>1$. Note that (ii) is equivalent to

(ii') there exists $z^{*}$ such that $\left|z^{*}\right|<1$ and $\left|h^{\prime}\left(z^{*}\right)\right|>1$, where

$$
h(z)=\frac{f\left(\tilde{z}+\left(z_{1}-z_{2}\right) z\right)}{\left(z_{1}-z_{2}\right)^{k}} .
$$

Since $\left|h^{\prime}(z)\right| \geq h^{\#}(z)$, it suffices to have $h^{\#}\left(z^{*}\right)>1$ in $\left(\mathrm{ii}^{\prime}\right)$.

Our next result deals with the situation in which the functions $f_{n}$ have more than a single zero in each neighborhood of a point of non-normality.

LEMMA 6. Let $\left\{f_{n}\right\}$ be a sequence of functions meromorphic on $\Delta$, all of whose zeros have multiplicity at least $k+1$, such that $f_{n}^{(k)}(z) \neq 1$ for all $n$ and all $z \in \Delta$. Suppose that 
(a) no subsequence of $\left\{f_{n}\right\}$ is normal at $z_{0}$, and

(b) for each $\delta>0, f_{n}$ has at least two distinct zeros on $\Delta\left(z_{0}, \delta\right)$ for sufficiently large $n$.

Then for each $\delta>0, f_{n}$ has a k-nontrivial pair $\left(a_{n}, c_{n}\right)$ of zeros on $\Delta\left(z_{0}, \delta\right)$ for sufficiently large $n$, and

$$
\left\{\frac{f_{n}\left(d_{n}+\left(a_{n}-c_{n}\right) \zeta\right)}{\left(a_{n}-c_{n}\right)^{k}}\right\}
$$

is not normal on $\Delta$. Here $d_{n}=\left(a_{n}+c_{n}\right) / 2$.

Proof. As in the proof of the previous lemma, it follows from (a) and Lemmas 1 and 4 that for each subsequence of $\left\{f_{n}\right\}$ there exists a (sub)subsequence (which, renumbering, we continue to denote by $\left\{f_{n}\right\}$ ), points $z_{n} \rightarrow z_{0}$, numbers $\rho_{n} \rightarrow 0^{+}$, and distinct $a, b \in \boldsymbol{C}$ such that

$$
g_{n}(\zeta)=\frac{f_{n}\left(z_{n}+\rho_{n} \zeta\right)}{\rho_{n}^{k}} \stackrel{\chi}{\Rightarrow} g(\zeta)=\frac{1}{k !} \frac{(\zeta-a)^{k+1}}{\zeta-b} \quad \text { on } \boldsymbol{C} .
$$

Thus there exist $\xi_{n} \rightarrow a, \eta_{n} \rightarrow b$ so that $a_{n}=z_{n}+\rho_{n} \xi_{n} \rightarrow z_{0}, b_{n}=z_{n}+\rho_{n} \eta_{n} \rightarrow z_{0}$ and $g_{n}\left(\xi_{n}\right)=f_{n}\left(a_{n}\right)=0, g_{n}\left(\eta_{n}\right)=f_{n}\left(b_{n}\right)=\infty$ for $n$ sufficiently large.

By assumption, there also exists $c_{n} \neq a_{n}, c_{n} \rightarrow z_{0}$, such that $f_{n}\left(c_{n}\right)=0$. Thus $c_{n}=z_{n}+\rho_{n} \xi_{n}^{*}$ and $\xi_{n}^{*} \rightarrow \infty$ by (6). Setting $d_{n}=\left(a_{n}+c_{n}\right) / 2$, we see that the function

$$
h_{n}(\zeta)=\frac{f_{n}\left(d_{n}+\left(a_{n}-c_{n}\right) \zeta\right)}{\left(a_{n}-c_{n}\right)^{k}}
$$

is defined for any $\zeta \in \boldsymbol{C}$ if $n$ is sufficiently large. We claim that $\left\{h_{n}\right\}$ is not normal at $\zeta=1 / 2$. Indeed, we have

$$
\begin{array}{rlrl}
\frac{a_{n}-d_{n}}{a_{n}-c_{n}} & \rightarrow \frac{1}{2}, & \frac{b_{n}-d_{n}}{a_{n}-c_{n}} \rightarrow \frac{1}{2}, \\
h_{n}\left(\frac{a_{n}-d_{n}}{a_{n}-c_{n}}\right)=f_{n}\left(a_{n}\right) & =0, & & h_{n}\left(\frac{b_{n}-d_{n}}{a_{n}-c_{n}}\right)=f_{n}\left(b_{n}\right)=\infty,
\end{array}
$$

so $\left\{h_{n}\right\}$ fails to be equicontinuous in a neighborhood of $1 / 2$. It follows from Marty's Theorem that

$$
\lim _{n \rightarrow \infty} \sup _{|\zeta-1 / 2| \leq 1 / 4} h_{n}^{\#}(\zeta)=\infty .
$$

Thus $\left(a_{n}, c_{n}\right)$ is a $k$-nontrivial pair of zeros of $f_{n}$ for $n$ sufficiently large.

LEMMA 7. Let $\left\{f_{n}\right\}$ be a sequence of functions meromorphic on $\Delta$, all of whose zeros have multiplicity at least $k+1$, such that $f_{n}^{(k)}(z) \neq 1$ for all $n$ and all $z \in \Delta$. Suppose that 
(a) there exist $d \in \Delta, a_{n} \rightarrow d, c_{n} \rightarrow d$, and $z_{0} \in C$ such that for every $\delta>0$,

$$
h_{n}(z)=\frac{f_{n}\left(d_{n}+\left(a_{n}-c_{n}\right) z\right)}{\left(a_{n}-c_{n}\right)^{k}}
$$

has at least two distinct zeros on $\Delta\left(z_{0}, \delta\right)$ for sufficiently large $n$, where $d_{n}=\left(a_{n}+c_{n}\right) / 2 ;$ and

(b) no subsequence of $\left\{h_{n}\right\}$ is normal at $z_{0}$.

Then for $n$ sufficiently large, $f_{n}$ has a $k$-nontrivial pair of zeros $\left(z_{n, 1}^{*}, z_{n, 2}^{*}\right)$ such that $z_{n, j}^{*} \rightarrow d(j=1,2)$ and $\left|z_{n, 1}^{*}-z_{n, 2}^{*}\right|<\left|a_{n}-c_{n}\right|$.

Proof. As before, it follows from Lemmas 1 and 4 that to each subsequence of $\left\{h_{n}\right\}$ there corresponds a subsequence (which we continue to write as $\left\{h_{n}\right\}$ ), $z_{n} \rightarrow z_{0}$, and $\rho_{n} \rightarrow 0^{+}$such that

$$
g_{n}(\zeta)=\frac{h_{n}\left(z_{n}+\rho_{n} \zeta\right)}{\rho_{n}^{k}} \stackrel{\chi}{\Rightarrow} \frac{1}{k !} \frac{(\zeta-a)^{k+1}}{\zeta-b} \text { on } \boldsymbol{C} .
$$

Thus there exist $\xi_{n, 0} \rightarrow b, \xi_{n, 1} \rightarrow a$ so that $z_{n, j}=z_{n}+\rho_{n} \xi_{n, j} \rightarrow z_{0}(j=0,1)$ and $g_{n}\left(\xi_{n, 0}\right)=h_{n}\left(z_{n, 0}\right)=\infty, g_{n}\left(\xi_{n, 1}\right)=h_{n}\left(z_{n, 1}\right)=0$. By (a), there exist $z_{n, 2} \rightarrow z_{0}$, $z_{n, 2} \neq z_{n, 1}$, such that $h_{n}\left(z_{n, 2}\right)=0$. Setting $z_{n, 2}=z_{n}+\rho_{n} \xi_{n, 2}$, we have $\xi_{n, 2} \rightarrow \infty$. Now put

$$
z_{n, j}^{*}=d_{n}+\left(a_{n}-c_{n}\right) z_{n}+\rho_{n}\left(a_{n}-c_{n}\right) \xi_{n, j} \quad j=0,1,2 .
$$

Clearly $z_{n, j}^{*} \rightarrow d, j=0,1,2$. Define

$$
G_{n}(\zeta)=\frac{f_{n}\left(\left(z_{n, 1}^{*}+z_{n, 2}^{*}\right) / 2+\left(z_{n, 1}^{*}-z_{n, 2}^{*}\right) \zeta\right)}{\left(z_{n, 1}^{*}-z_{n, 2}^{*}\right)^{k}} .
$$

Then $\left\{G_{n}\right\}$ is not normal at $\zeta=1 / 2$. Indeed,

$$
G_{n}\left(\frac{2 \xi_{n, 0}-\xi_{n, 1}-\xi_{n, 2}}{2\left(\xi_{n, 1}-\xi_{n, 2}\right)}\right)=\infty, \quad G_{n}(1 / 2)=0 .
$$

Since $\left(2 \xi_{n, 0}-\xi_{n, 1}-\xi_{n, 2}\right) / 2\left(\xi_{n, 1}-\xi_{n, 2}\right) \rightarrow 1 / 2, \quad\left\{G_{n}\right\}$ is not equicontinuous at $\zeta=1 / 2$. As before, it follows from Marty's Theorem that $\left(z_{n, 1}^{*}, z_{n, 2}^{*}\right)$ is a $k$ nontrivial pair of zeros of $f_{n}$. Now $\left|z_{n, 1}^{*}-z_{n, 2}^{*}\right|=\left|a_{n}-c_{n}\right|\left|z_{n, 1}-z_{n, 2}\right|$; therefore, since $z_{n, j} \rightarrow z_{0}(j=1,2)$, we have $\left|z_{n, 1}^{*}-z_{n, 2}^{*}\right|<\left|a_{n}-c_{n}\right|$ for large enough $n$, as required.

LEMMA 8. Let $\left\{f_{n}\right\}$ be a sequence of functions meromorphic on $\Delta$, all of whose zeros have multiplicity at least $k+1$, such that $f_{n}^{(k)}(z) \neq 1$ for all $n$ and all $z \in \Delta$. Suppose that

(a) $\left\{f_{n}\right\}$ is normal on $\Delta^{\prime}(0,1)$, but no subsequence of $\left\{f_{n}\right\}$ is normal at 0 ; and

(b) there exists $\delta>0$ such that $f_{n}$ has a single (multiple) zero on $\Delta(0, \delta)$ for all sufficiently large $n$.

Then there exists a subsequence of $\left\{f_{n}\right\}$ (which we continue to call $\left\{f_{n}\right\}$ ) such that for any $a \in \boldsymbol{C}, f_{n}-a$ has at most $k+1$ zeros (counting multiplicity) on $\Delta(0,1 / 2)$. 
Proof. Taking a subsequence and renumbering, we may assume that $f_{n} \stackrel{\chi}{\Rightarrow} f$ on $\Delta^{\prime}(0,1)$. By Lemma $5, f(z)=z^{k} / k !$. Suppose that $|a|<(2 / 3)^{k} / k !$. Taking $\Gamma$ to be the circle $\{|z|=3 / 4\}$ traversed once in the positive direction, we have

$$
\frac{1}{2 \pi i} \int_{\Gamma} \frac{f_{n}^{\prime}(z)}{f_{n}(z)-a} d z \rightarrow \frac{1}{2 \pi i} \int_{\Gamma} \frac{z^{k-1} /(k-1) !}{z^{k} / k !-a} d z=k .
$$

However, the left hand side is the number of $a$-points of $f_{n}$ minus the number of poles of $f_{n}$ inside $\Gamma$, counting multiplicities. By Lemma 5, there exists $0<\delta<$ $3 / 4$ such that $f_{n}$ has a single simple pole on $\Delta(0, \delta)$ for $n$ sufficiently large. Since $f_{n}$ converges uniformly to $z^{k} / k !$ on $\{z: \delta \leq|z| \leq 3 / 4\}$, there exists $N_{1}$ such that if $n \geq N_{1} f_{n}$ has a single simple pole in $\Delta(0,3 / 4)$. Hence for $n \geq N_{1}, f_{n}$ takes on the value $a$ (counting multiplicities) exactly $k+1$ times on $\Delta(0,3 / 4)$.

Suppose now that $|a|>(2 / 3)^{k} / k$ !. Let $\Gamma^{\prime}$ be the circle $\{|z|=5 / 9\}$ traversed in the positive direction. Then

$$
\frac{1}{2 \pi i} \int_{\Gamma^{\prime}} \frac{f_{n}^{\prime}(z)}{f_{n}(z)-a} d z \rightarrow \frac{1}{2 \pi i} \int_{\Gamma^{\prime}} \frac{z^{k-1} /(k-1) !}{z^{k} / k !-a} d z=0,
$$

so the number of $a$-points minus the number of poles of $f_{n}$ (counting multiplicity) inside $\Gamma^{\prime}$ is 0 for large $n$. It follows as before that there exists $N_{2}$ such that $f_{n}$ takes on the value $a$ exactly once (counting multiplicities) on $\Delta(0,5 / 9)$ if $n \geq N_{2}$. Dropping the elements $f_{n}$ with $n<\max \left(N_{1}, N_{2}\right)$ and renumbering, we obtain the desired sequence.

Lemma 9. Let $f$ be a meromorphic function on $\boldsymbol{C}$, all of whose zeros have multiplicity at least $k+1$, such that $f^{(k)}(z) \neq 1, z \in C$. Then either

(i) $f$ is rational; or

(ii) there exist k-nontrivial pairs $\left(a_{n}, c_{n}\right)$ of zeros of $f$ such that $\left|a_{n}-c_{n}\right| \rightarrow 0$ and a sequence of functions

$$
h_{n}(\zeta)=\frac{f\left(d_{n}+\left(a_{n}-c_{n}\right) \zeta\right)}{\left(a_{n}-c_{n}\right)^{k}}
$$

which is not normal on $\Delta$; here $d_{n}=\left(a_{n}+c_{n}\right) / 2$.

Proof. Suppose $f$ is not rational. Then by Lemma $4, f$ has infinite order, so there exist $z_{n} \rightarrow \infty$ and $\varepsilon_{n} \rightarrow 0$ such that

$$
S\left(\Delta\left(z_{n}, \varepsilon_{n}\right), f\right)=\frac{1}{\pi} \iint_{\left|z-z_{n}\right| \leq \varepsilon_{n}}\left[f^{\#}(z)\right]^{2} d x d y \rightarrow \infty .
$$

Indeed, otherwise there would exist $\varepsilon>0$ and $M>0$ such that $S(\Delta(\zeta, \varepsilon), f) \leq M$ for all $\zeta \in \boldsymbol{C}$. From this follows

$$
S(r)=\frac{1}{\pi} \iint_{|z|<r}\left[f^{\#}(z)\right]^{2} d x d y=O\left(r^{2}\right),
$$


so that (cf. [10, p. 217]) $f$ would have order at most 2, a contradiction. In particular, there exist $z_{n}^{*} \in \Delta\left(z_{n}, \varepsilon_{n}\right)$ such that $f^{\#}\left(z_{n}^{*}\right) \rightarrow \infty$. Let $f_{n}(z)=$ $f\left(z+z_{n}^{*}\right)$. Then no subsequence of $\left\{f_{n}\right\}$ is normal at 0 .

Suppose there exists $\delta>0$ such that $f_{n}$ has only a single (multiple) zero $\xi_{n}$ on $\Delta(0, \delta)$. Since no subsequence of $\left\{f_{n}\right\}$ is normal at $0, \xi_{n} \rightarrow 0$ by Lemma 2 . Thus, again by Lemma $2,\left\{f_{n}\right\}$ is normal on $\Delta^{\prime}(0, \delta)$. It follows from Lemma 8 that there exist $n_{1}<n_{2}<\cdots$ such that for any $a \in C, f_{n_{j}}-a$ has at most $k+1$ zeros (counting multiplicity) on $\Delta(0, \delta / 2)$. Thus, for large enough $j$,

$$
S\left(\Delta\left(z_{n_{j}}, \varepsilon_{n_{j}}\right), f\right) \leq S\left(\Delta(0, \delta / 2), f_{n_{j}}\right) \leq k+1
$$

which contradicts $(7)$.

Thus, for each $\delta>0, f_{n}$ has at least two distinct zeros on $\Delta(0, \delta)$ for sufficiently large $n$. The result now follows immediately from Lemma 6 .

\section{Proof of the Theorem}

Suppose the Theorem is false. Then there exists a sequence $\left\{a_{j}^{*}\right\} \subset D$ with no accumulation point in $D$ and such that $a_{1}^{*} \neq a_{2}^{*}$ and a sequence $\left\{f_{n}\right\} \subset \mathscr{F}$ such that $f_{n} \stackrel{\chi}{\Rightarrow} f$ on $D \backslash\left\{a_{j}^{*}\right\}$ but no subsequence of $\left\{f_{n}\right\}$ is normal at $a_{1}^{*}$ or $a_{2}^{*}$. We may assume that $a_{1}^{*}=0$ and $D=\Delta$. The argument given in the proof of Lemma 5 shows that $f_{n}^{(k)} \Rightarrow 1$ on $\Delta \backslash\left\{a_{j}^{*}\right\}$ or $f=\infty$, so $f \not \equiv 0$.

If there exists $\delta>0$ such that $f_{n}$ has only a single (multiple) zero on each $\Delta\left(a_{j}^{*}, \delta\right)(j=1,2)$ for large enough $n$, it follows from Lemma 5 that $f(z)=$ $\left(z-a_{j}^{*}\right)^{k} / k !(j=1,2)$ on $\Delta \backslash\left\{a_{j}^{*}\right\}$. Thus $a_{1}^{*}=a_{2}^{*}$, a contradiction.

Therefore, one may suppose that for any $\delta>0, f_{n}$ has at least two distinct zeros on $\Delta(0, \delta)$ for sufficiently large $n$. By Lemma $6, f_{n}$ has a $k$-nontrivial pair of zeros in $\Delta(0, \delta)$ for $n$ large enough. Therefore, some subsequence of $\left\{f_{n}\right\}$ (which, as usual, we continue to call $\left\{f_{n}\right\}$ ) has a $k$-nontrivial pair of zeros $\left(z_{n}, w_{n}\right)$ such that $\left|z_{n}\right|<1 / n,\left|w_{n}\right|<1 / n$. There exist $\delta_{0}>0$ and $1<s<2$ such that $f_{n} \stackrel{\chi}{\Rightarrow} f$ on $\Delta^{\prime}\left(0,2 \delta_{0}\right)$ and $f$ does not vanish for $\delta_{0} \leq|z| \leq s \delta_{0}$. For $1 / n<\delta_{0}$, let $\left(a_{n}, c_{n}\right)$ be a $k$-nontrivial pair of zeros of $f_{n}$ in $\Delta\left(0, \delta_{0}\right)$ whose distance is minimal. Clearly, $a_{n}-c_{n} \rightarrow 0$. Set $d_{n}=\left(a_{n}+c_{n}\right) / 2$. Then $d_{n} \in \Delta\left(0, \delta_{0}\right)$; and, passing to a subsequence, we may assume that $d_{n} \rightarrow a$, so $|a| \leq \delta_{0}$. Since $f$ and $f_{n}$ have no zeros on $\left\{z: \delta_{0} \leq|z| \leq s \delta_{0}\right\}$ if $n$ is large enough, $\left(a_{n}, c_{n}\right)$ is a $k$-nontrivial pair of zeros of $f_{n}$ on $\Delta\left(0, s \delta_{0}\right)$ whose distance is minimal.

Set

$$
h_{n}(\zeta)=\frac{f_{n}\left(d_{n}+\left(a_{n}-c_{n}\right) \zeta\right)}{\left(a_{n}-c_{n}\right)^{k}} .
$$

Then for each $\zeta \in \boldsymbol{C}, h_{n}(\zeta)$ is defined if $n$ is sufficiently large. Clearly, all zeros of $h_{n}$ have multiplicity at least $k+1$ and $h_{n}^{(k)}(\zeta) \neq 1$. We claim that no subsequence of $\left\{h_{n}\right\}$ is normal on $\boldsymbol{C}$. Otherwise, taking a subsequence and renumbering, we would have $h_{n} \stackrel{\chi}{\Rightarrow} h$ on $\boldsymbol{C}$. Since $\left(a_{n}, c_{n}\right)$ is a $k$-nontrivial pair of zeros of $f_{n}, h_{n}( \pm 1 / 2)=h_{n}^{\prime}( \pm 1 / 2)=\cdots=h_{n}^{(k)}( \pm 1 / 2)=0$, and $\sup _{\Delta}\left|h_{n}^{(k)}(z)\right|>1$. 
It follows easily that $h^{(k)}(\zeta) \neq 1$ on $\boldsymbol{C}$ and that $h$ is nonconstant. Since all zeros of $h$ have multiplicity at least $k+1$, Lemma 4 shows that $h$ must be transcendental. It then follows from Lemma 9 that there exist infinitely many $k$ nontrivial pairs $\left(\xi_{j}, \eta_{j}\right)$ of zeros of $h$ such that $\xi_{j} \rightarrow \infty$ and $\xi_{j}-\eta_{j} \rightarrow 0$, and $z_{j}^{*}$ with $\left|z_{j}^{*}-\left(\xi_{j}+\eta_{j}\right) / 2\right|<\left|\xi_{j}-\eta_{j}\right|$ and $h^{\#}\left(z_{j}^{*}\right) \rightarrow \infty$.

Fix $j$ such that $h^{\#}\left(z_{j}^{*}\right) \geq 2$ and $\left|\xi_{j}-\eta_{j}\right|<1$. Then there exist $\xi_{n, j} \rightarrow \xi_{j}$ and $\eta_{n, j} \rightarrow \eta_{j}$ such that for $n$ sufficiently large, $h_{n}\left(\xi_{n, j}\right)=h_{n}\left(\eta_{n, j}\right)=0$ and $\left|z_{j}^{*}-\left(\xi_{n, j}+\eta_{n, j}\right) / 2\right|<\left|\xi_{n, j}-\eta_{n, j}\right|$. Put

$$
\xi_{n, j}^{*}=d_{n}+\left(a_{n}-c_{n}\right) \xi_{n, j} \quad \eta_{n, j}^{*}=d_{n}+\left(a_{n}-c_{n}\right) \eta_{n, j} \quad z_{n, j}^{*}=d_{n}+\left(a_{n}-c_{n}\right) z_{j}^{*} .
$$

Then

$$
\left|z_{n, j}^{*}-\frac{\xi_{n, j}^{*}+\eta_{n, j}^{*}}{2}\right|=\left|a_{n}-c_{n}\right|\left|z_{j}^{*}-\frac{\xi_{n, j}+\eta_{n, j}}{2}\right|<\left|a_{n}-c_{n}\right|\left|\xi_{n, j}-\eta_{n, j}\right|=\left|\xi_{n, j}^{*}-\eta_{n, j}^{*}\right|,
$$

where $\xi_{n, j}^{*} \rightarrow a, \quad \eta_{n, j}^{*} \rightarrow a$ and $|a|<s \delta_{0}$; also, for $n$ sufficiently large, $\left|f_{n}^{\prime}\left(z_{n, j}^{*}\right) /\left(a_{n}-c_{n}\right)^{k-1}\right|=\left|h_{n}^{\prime}\left(z_{j}^{*}\right)\right| \geq h_{n}^{\#}\left(z_{j}^{*}\right)>1$. We conclude that $\left(\xi_{n, j}^{*}, \eta_{n, j}^{*}\right)$ is a $k$-nontrivial pair of zeros of $f_{n}$ on $\Delta\left(0, s \delta_{0}\right)$. However,

$$
\left|\xi_{n, j}^{*}-\eta_{n, j}^{*}\right|=\left|a_{n}-c_{n}\right|\left|\xi_{n, j}-\eta_{n, j}\right|<\left|a_{n}-c_{n}\right|
$$

if $n$ is sufficiently large. This contradicts the fact that $\left(a_{n}, c_{n}\right)$ is a $k$-nontrivial pair of zeros of $f_{n}$ in $\Delta\left(0, s \delta_{0}\right)$ whose distance is minimal.

Thus no subsequence of $\left\{h_{n}\right\}$ is normal on $C$. Let $E$ be the set on which $\left\{h_{n}\right\}$ is not normal. Suppose that for each $\zeta \in E$, there is a neighborhood on which $h_{n}$ has only a single (multiple) zero for sufficiently large $n$. Then by Lemma $2,\left\{h_{n}\right\}$ is quasinormal at each point of $E$ and hence on all of $\boldsymbol{C}$. Let $\zeta_{0} \in E$. Taking a subsequence, we may assume that no subsequence of $\left\{h_{n}\right\}$ is normal at $\zeta_{0}$ and that $\left\{h_{n}\right\}$ converges locally spherically uniformly on $\boldsymbol{C} \backslash E_{0}$, where $E_{0} \subset E$ is a discrete set containing $\zeta_{0}$. By Lemma $5, h_{n} \stackrel{\chi}{\Rightarrow}\left(\zeta-\zeta_{0}\right)^{k} / k$ ! on $C \backslash E_{0}$. Taking additional subsequences and diagonalizing, we may assume that no subsequence of $\left\{h_{n}\right\}$ is normal at any point of $E_{0}$. We claim that $E_{0}=\left\{\zeta_{0}\right\}$. Indeed, otherwise there exists $\zeta_{1} \in E_{0}, \zeta_{1} \neq \zeta_{0}$; then, as before, it follows from Lemma 5 that $h_{n}(\zeta) \stackrel{\chi}{\Rightarrow}\left(\zeta-\zeta_{1}\right)^{k} / k$ ! on $\boldsymbol{C} \backslash E_{0}$, so that $\zeta_{1}=\zeta_{0}, E_{0}=\left\{\zeta_{0}\right\}$, and $h_{n}(\zeta) \stackrel{\chi}{\Rightarrow}\left(\zeta-\zeta_{0}\right)^{k} / k$ ! on $C \backslash\left\{\zeta_{0}\right\}$. But this contradicts $h_{n}( \pm 1 / 2)=0$. Hence there exists $\zeta_{0} \in E$ such that for each $\delta>0$, there is a subsequence of $\left\{h_{n}\right\}$ (which we continue to call $\left\{h_{n}\right\}$ ) such that each $h_{n}$ has at least two distinct zeros in $\Delta\left(\zeta_{0}, \delta\right)$ for sufficiently large $n$. Then by Lemma 7 , for $n$ sufficiently large, $f_{n}$ has a nontrivial pair of zeros $\left(w_{n, 1}^{*}, w_{n, 2}^{*}\right)$ such that $w_{n, j}^{*} \rightarrow a(j=1,2)$ and $\left|w_{n, 1}^{*}-w_{n, 2}^{*}\right|<\left|a_{n}-c_{n}\right|$. This contradicts the fact that $\left(a_{n}, c_{n}\right)$ is a nontrivial pair of zeros of $f_{n}$ in $\Delta\left(0, s \delta_{0}\right)$ whose distance is minimal.

Acknowledgment. We would like to thank L. Zalcman for fruitful discussions and useful advice. 


\section{REFERENCES}

[1] Walter Bergweiler and J. K. Langley, Multiplicities in Hayman's alternative, preprint.

[2] Chi-Tai Chuang, Normal Families of Meromorphic Functions, World Scientific, 1993.

[3] Ku Yongxing, Un critère de normalité des familles de fonctions méromorphes, Sci. Sinica Special Issue 1 (1979), 267-274 (Chinese).

[4] Shahar Nevo, On theorems of Yang and Schwick, Complex Variables 46 (2001), 315-321.

[5] Shahar Nevo, Applications of Zalcman's lemma to $Q_{m}$-normal families, Analysis 21 (2001), 289-325.

[6] Xuecheng Pang, Shahar Nevo, and Lawrence Zalcman, Quasinormal families of meromorphic functions, Rev. Mat. Iberoamericana, to appear.

[ 7] Xuecheng Pang and Lawrence Zalcman, Normal families and shared values, Bull. London Math. Soc 32 (2000), 325-331.

[ 8 ] Xuecheng Pang and Lawrence Zalcman, Normal families of meromorphic functions with multiple zeros and poles, Israel J. Math. 136 (2003), 1-9.

[9] Yufei Wang and Mingliang Fang, Picard values and normal families of meromorphic functions with multiple zeros, Acta Math. Sinica N.S. 14 (1998), 17-26.

[10] Lawrence Zalcman, Normal families: new perspectives, Bull. Amer. Math. Soc. 35 (1998), 215-230.

Department of Mathematics and Statistics

BAR-ILAN UNIVERSITY

52900 RAMAT-GAN

ISRAEL

e-mail: nevosh@macs.biu.ac.il

Department of Mathematics

East China Normal University

SHANGHAI 200062

P. R. CHINA

e-mail: xcpang@math.ecnu.edu.cn 